\title{
Potentiometric Determination of Dopamine in Pharmaceutical Preparations by Crown Ether-PVC Membrane Sensors
}

\author{
A. M. Othman, * N. M. H. Rizka,* and M. S. El-Shahawi**ं \\ *Engineering and Biotechnology Research Institute (GEBRI), Minufiya University, Sadat City, Egypt \\ **Chemistry Department, Faculty of Science at Damietta, Mansoura University, Damietta, Egypt
}

\begin{abstract}
Two simple, rapid and sensitive sensors for the assay of dopamine hydrochloride have been developed. The methods are based upon the formation of the membrane sensors 12-crown-4-phosphotungestic acid (crown ether-PTA)-dopamine and 12-crown-4-tetraphenylborate (crown ether-TPB)-dopamine as neutral carriers. The sensors were stable and showed fast potential responses of $10 \mathrm{~s}$, and near-Nernstian cationic slopes of $53.3-56.2 \mathrm{mV} /$ decade of activity between $\mathrm{pH} 2.2-6$ for the monovalent dopamine cation over a wide range concentrations $1 \times 10^{-5}-1 \times 10^{-1} \mathrm{M}$. The selectivity coefficients of the developed sensors indicated excellent selectivity for dopamine over a large number of organic and inorganic species and pharmaceutical excipients. The mediator $o$-nitrophenyloctyl ether significantly affected the lifetime of the fabricated sensors of dopamine. Satisfactory results were obtained for the determination of dopamine in dosage form by the proposed sensors with an average recovery of $99.85 \%$ for the nominal concentration.
\end{abstract}

(Received June 2, 2003; Accepted November 25, 2003)

Dopamine (DA) is one of the naturally occurring catecholamines, 4-(2-aminoethyl)benzene-1,2-diol hydrochloride (Fig. 1). It is an important chemical transmitter and an adrenal modular hormone at the terminals of sympathetic nerve. ${ }^{1}$ Dopamine also acts as a biogenic amine, which functions as a neurotransmitter in the brain and nervous system of mammals. ${ }^{2}$ Dopamine hydrochloride salt is widely used in the treatment of bronchial asthma, hypertension, heart failure, cardiac surgery and renal failures associated with shock episodes. ${ }^{3,4}$

The detection and quantification of dopamine are of practical importance in chemical, biological, clinical, environmental and many other fields. A large number of procedures, e.g. spectrophotometric, ${ }^{5-8}$ gas, liquid and high-performance liquid chromatography, ${ }^{9-13}$ potentiometry and voltammetric methods ${ }^{14-16}$ have been developed. A number of biosensors at carbon-paste electrode modified with polyphenol oxidase have been fabricated for dopamine assay in the presence of metabolically related compounds. ${ }^{16}$ The present paper describes the use of two novel sensors for the analysis of dopamine hydrochloride in pharmaceutical products. The two sensors are based upon the use of an ion-association complex of 12-crown4-tetraphenylborate or 12-crown-4-phosphotungestic acid as a neutral carrier in a plasticized PVC-matrix with a solvent mediator, o-nitrophenyloctyl ether. The two sensors display stable, fast and linear response over a wide range of concentrations and $\mathrm{pH}$ values.

\section{Experimental}

\section{Chemicals and materials}

All of the chemicals and reagents were of analytical reagent grade unless otherwise stated. Doubly distilled water was used to prepare stock $(0.1 \mathrm{M})$ and dilute solutions of dopamine

$\doteqdot$ To whom correspondence should be addressed. hydrochloride, excipients, diluents and metal chloride or nitrate salts. Low-molecular-weight polyvinyl chloride (PVC), $O$ nitrophenyloctyl ether (o-NPOE), sodium tetraphenyl borate (NaTPB), phosphotungestic acid (PTA) and dopamine hydrochloride (DA) were supplied from Sigma. The crown ether (CE) 12-crown-4-ether, namely 1,4,7,10,13pentaoxacyclopentadecane, and tetrahydrofuran (THF) were obtained from Fluka. Pharmaceutical preparations of dopamine<smiles>[NH3+]CCc1ccc(O)c(O)c1</smiles><smiles>NCCc1ccc(O)c(O)c1</smiles>

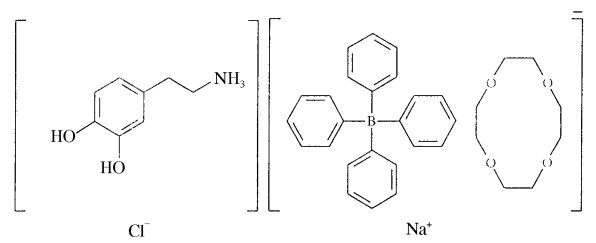

Fig. 1 Chemical structures of dopamine (A), CE-PTA (B) and CETPB (C). 
hydrochloride were purchased from local drug stores and were of USP quality. A standard stock solution $\left(1 \times 10^{-1} \mathrm{M}\right)$ of dopamine hydrochloride was prepared by dissolving $0.474 \mathrm{~g}$ of the salt in $25 \mathrm{ml}$ acetate buffer ( $\mathrm{pH} 4$ ). Dilute solutions of the drug $10^{-2}-10^{-6} \mathrm{M}$ were freshly prepared by diluting the stock solution with doubly distilled water.

\section{Apparatus}

Potentiometric measurements were carried out at $25 \pm 1{ }^{\circ} \mathrm{C}$ with an Orion $\mathrm{pH} / \mathrm{mV}$ meter (Model 720) microprocessor Ionalyzer. Two sensors, dopamine-12-crown-4-tetraphenylborate and dopamine-12-crown-4-phosphotungestic acid-PVC membrane sensors, were used with a double-junction $\mathrm{Ag} / \mathrm{AgCl}$ reference electrode (Orion Model 90-02) containing a 10\% (w/v) sodium nitrate solution in the outer compartment. The $\mathrm{pH}$ measurements were performed with a combination Ross glass electrode (Orion Model 81-02). A Perkin-Elmer Lambada 15 UV/VIS spectrophotometer was used for absorbance measurements.

\section{Procedures}

Preparation of ionophores. The ion-association complexes (ionophores), 12-crown-4-phosphotungestic acid (CE-PTA) and 12-crown-4-tetraphenylborate (CE-TPB), were prepared by mixing $10 \mathrm{ml}$ of $1 \times 10^{-2} \mathrm{M}$ of PMA or NaTPB with $10 \mathrm{ml}$ of $10^{-2} \mathrm{M}$ of 12-crown-4, respectively. The mixtures were then shaken well for $10 \mathrm{~min}$ and the produced precipitates were filtered off through Whatman filter paper (No. 42), washed with deionized water, dried at room temperature and finally ground to a fine powder.

Fabrication of PVC dopamine membrane sensors. A $10 \mathrm{mg}$ portion of the complex ion associate CE-PTA or CE-TPB was mixed in a glass petri dish (5 cm diameter) with $170 \mathrm{mg}$ of PVC powder and $380 \mathrm{mg}$ of the plasticizer $o$-nitorphenyloctyl ether. The cocktail was dissolved in $5 \mathrm{ml}$ of freshly distilled THF; and the petri dish was covered with filter paper and left overnight to allow slow evaporation of the solvent at room temperature. A transparent master PVC membrane with an average thickness of $\approx 0.1 \mathrm{~mm}$ was obtained, sectioned with a cork borer $(10 \mathrm{~mm}$ diameter) and glued to polyethylene tubing as previously described. ${ }^{18,19}$ The electrode was filled with an internal solution prepared from a mixture of an equal volume of an aqueous $\mathrm{Ag} / \mathrm{AgCl} / 10^{-2} \mathrm{M} \mathrm{KCl}-10^{-2} \mathrm{M}$ dopamine solution \| PVC membrane $\| 10^{-2} \mathrm{M}$ solution $\left(10^{-2} \mathrm{M}\right)$ dopamine at $\mathrm{pH} 4.0$ and potassium chloride. The prepared sensors were finally preconditioned by soaking them in a dopamine hydrochloride solution $\left(1 \times 10^{-2} \mathrm{M}\right)$ for $1 \mathrm{~h}$ and stored in this solution when not in use. A reference $\mathrm{Ag} / \mathrm{AgCl}$ wire electrode $(1 \mathrm{~mm}$ diameter) was immersed in the internal solution.

\section{Sensors calibrations}

The developed sensors were calibrated by transferring $10 \mathrm{ml}$ aliquots of an aqueous solution $\left(10^{-1}-10^{-6} \mathrm{M}\right)$ of dopamine hydrochloride to $50 \mathrm{ml}$ beakers, followed by immersing the dopamine-PVC membrane sensor in conjunction with a doublejunction $\mathrm{Ag} / \mathrm{AgCl}$ reference electrode in the test solution. The potential readings were recorded after stabilization to $0.2 \mathrm{mV}$, and the e.m.f was plotted as a function of the logarithmic of the dopamine concentration. The calibration graphs were used for subsequent determinations of the unknown dopamine concentrations.

\section{Sensor selectivity}

The potentiometric selectivity coefficients $\left(K_{\mathrm{DA}, \mathrm{B}}^{\mathrm{pot}}\right)$ of the two sensors were determined by a separate solution technique using
$1 \times 10^{-2} \mathrm{M}$ of the drug and the interferent ${ }^{18}$ as follows. The proposed sensor, CE-PTA or CE-PMA, was immersed in a 10 $\mathrm{ml}$ portion of $10^{-2} \mathrm{M}$ of a dopamine solution adjusted to $\mathrm{pH} 4.0$ and a potential reading was then made. The potential of a $10^{-2}$ $\mathrm{M}$ solution of the interferent species adjusted to $\mathrm{pH} 4$ was measured. The potentiometric selectivity coefficients $\left(K_{\mathrm{Ba}, \mathrm{B}}^{\mathrm{pot}}\right)$ is determined by employing the rearranged Niclosky equation, ${ }^{18,19}$

$$
\log K_{\mathrm{DA}, \mathrm{B}}^{\mathrm{pot}}=\left[E_{\mathrm{DA}}-E_{\mathrm{B}} / S\right]+\left[1+Z_{\mathrm{DA}} / Z_{\mathrm{B}}\right] \log D A
$$

where $E_{\mathrm{DA}}$ is the potential measured in $10^{-2} \mathrm{M}$ for dopamine, $E_{\mathrm{B}}$ is the potential measured in $10^{-2} \mathrm{M}$ of the interfering anion, $Z_{\mathrm{DA}}$ and $Z_{\mathrm{B}}$ are the charges of dopamine and the interfering ions, respectively, and $S$ is the slope of the electrode calibration curve plot. ${ }^{18}$

\section{Determination of dopamine in a pharmaceutical preparation}

Direct determination of dopamine hydrochloride. Four ampoules of each pharmaceutical product were well mixed by shaking and an appropriate volume $(5.0 \mathrm{ml})$ of an aliquot solution containing $1-10 \mathrm{mg}$ was accurately transferred to a $100 \mathrm{ml}$ calibrated flask and completed to the mark with acetate buffer ( $\mathrm{pH} 4$ ). The content was determined by measuring the e.m.f of the resulting solution by the proposed potentiometric method.

Standard addition method for the analysis of dopamine hydrochloride. Alternatively, the standard addition (spiking) technique was used as follows. A known volume of the test solution $(50 \mathrm{ml})$ of the drug at $\mathrm{pH} 4.0$ was transferred into a calibrated measuring flask $(100 \mathrm{ml})$. The potentials displayed by the test solution of the drug was measured before and after the addition of $0.5 \mathrm{ml}$ of the standard dopamine hydrochloride solution $\left(10^{-2} \mathrm{M}\right)$. The change in the electrode potential was then recorded and used for determining the drug. The proposed methods were then compared with the reported spectrophotometric method for dopamine determination in pharmaceutical preparations at room temperature. ${ }^{20}$

\section{Results and Discussion}

\section{Performance characteristics of dopamine sensors}

Macrocylic crown ethers are well-known as selective ligands for various ions. ${ }^{20-23}$ The compound 12-crown-4 reacted with phosphotungestic acid or tetraphenylborate, and the produced species were successfully used as neutral electrical carriers for the cationic response of some alkaline ions. ${ }^{22,23}$ These precipitates are easily prepared and have lower solubility than mono (crown ethers), and have similar characteristics as a crown ether. ${ }^{23}$ Dopamine reacts with phosphotungstic acid and tetraphenylborate, forming soluble ion-associate complexes in an aqueous medium. Thus, the precipitate CE-PTA or CE-TPB in a PVC membrane containing $o$-NPOE plasticizer was used as electrical carriers for the determination of dopamine hydrochloride after soaking in $10^{-2} \mathrm{M}$ DA solution. These membranes were electrochemically evaluated under a static mode of operation according to the IUPAC recommendation. ${ }^{24}$ The membranes were prepared using casting solutions of 30:2:68 wt $\%$ of polyvinyl chloride, neutral carrier complex and solvent mediator, respectively. Table 1 summarizes the response characteristics, e.g. calibration slopes, response time, detection limits and intervals the linearity, over a period of three months for different assemblies of each sensor at the optimum $\mathrm{pH}$ and room temperature using the IUPAC recommendations. ${ }^{24}$ The sensors showed good behavior regarding the response time 
Table 1 Performance characteristics of dopamine PVC membrane sensors

\begin{tabular}{lcc}
\hline \multirow{2}{*}{ Parameter $^{\mathrm{a}}$} & \multicolumn{2}{c}{ PVC membrane sensor } \\
\cline { 2 - 3 } & CE-PTA & CE-TPB \\
\hline Slope $(\mathrm{mV} /$ decade $)$ & $56.2 \pm 0.5$ & $53.3 \pm 0.54$ \\
Intercepte $(\mathrm{mV})$ & $-99 \pm 0.4$ & $-70 \pm 0.3$ \\
Correlation coefficient $(r)(n=5)$ & 0.997 & 0.998 \\
Lower limit of linear range $(\mathrm{M})$ & $6 \times 10^{-4}$ & $8 \times 10^{-4}$ \\
Lower limit of detection $(\mathrm{M})$ & $5 \times 10^{-5}$ & $6 \times 10^{-5}$ \\
Response time for $1 \times 10^{-3} \mathrm{M}(\mathrm{s})$ & 10 & 10 \\
Working pH range & $3.5-6.0$ & $2.5-6.0$ \\
\hline
\end{tabular}

a. Average of 5 measurements.

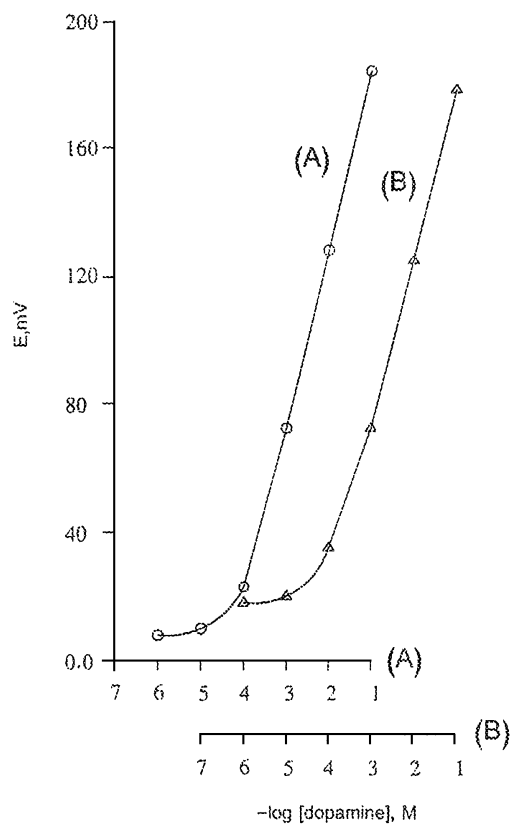

Fig. 2 Potentiometric responses of dopamine sensors CE-TPB (A) and CE-PTA (B).

and reproducibility of the e.m.f values of the electrodes. A typical calibration graph and the response characteristics of dopamine sensors based on CE-PTA or CE-TPB are shown in Fig. 2. At room temperature the two sensors displayed a linear response for dopamine hydrochloride over the concentration range $10^{-1}-10^{-6} \mathrm{M}$ and calibration slopes of $56.3 \mathrm{mV} /$ decade and $53.3 \mathrm{mV} /$ decade for the CE-PTA and CE-TPB sensors, respectively. The response time $\left(t_{95}\right)$ of dopamine sensors was tested by measuring the time required to achieve a $95 \%$ steady potential for $10^{-4}$ and $10^{-3} \mathrm{M}$ dopamine hydrochloride solutions when their concentrations were rapidly increased by one decade. Short response times of $10 \mathrm{~s}$ for DA $>10^{-3} \mathrm{M}$ and $30 \mathrm{~s}$ for DA > $10^{-4} \mathrm{M}$, were obtained. The sensors displayed constant potential readings within $1 \mathrm{mV}$ from day to day and the calibration slopes did not change by more than $1 \mathrm{mV}$ per decade over a period of two weeks for PVC sensors. All of the sensors exhibited constant-slope values during at least 6 weeks, followed by a gradual decrease in their sensitivity as time passed. The calibration graphs for the two electrodes were found to be reproducible from day to day, provided that the sensors were stored in appropriate dopamine before use.

It is well-known that crown ethers are very sensitive to the
Table 2 Selectivity coefficient $\left(K_{\mathrm{DA}, \mathrm{B}}^{\mathrm{pot}}\right)$ of dopamine PVC membrane sensors

\begin{tabular}{lcc}
\hline \multirow{2}{*}{ Interferent, B } & \multicolumn{2}{c}{$K_{\mathrm{DA}, \mathrm{B}}^{\mathrm{pot}}$} \\
\cline { 2 - 3 } & $\mathrm{CE}-\mathrm{PTA}$ & $\mathrm{CE}-\mathrm{TPB}$ \\
\hline L-Dopa & $3.16 \times 10^{-2}$ & $5.81 \times 10^{-2}$ \\
$\mathrm{Na}^{+}$ & $8.62 \times 10^{-2}$ & $1.39 \times 10^{-3}$ \\
$\mathrm{~K}^{+}$ & $1.00 \times 10^{-3}$ & $2.65 \times 10^{-3}$ \\
$\mathrm{Mg}^{2+}$ & $5.26 \times 10^{-3}$ & $2.34 \times 10^{-3}$ \\
$\mathrm{Ca}^{2+}$ & $1.26 \times 10^{-2}$ & $4.28 \times 10^{-2}$ \\
Urea & $2.18 \times 10^{-3}$ & $1.03 \times 10^{-2}$ \\
Triethanol amine & $1.95 \times 10^{-2}$ & $1.07 \times 10^{-2}$ \\
Glycine & $1.96 \times 10^{-3}$ & $1.15 \times 10^{-2}$ \\
Glucose & $5.38 \times 10^{-2}$ & $1.18 \times 10^{-2}$ \\
Maltose & $1.34 \times 10^{-3}$ & $4.86 \times 10^{-2}$ \\
Catechol & $2.31 \times 10^{-3}$ & $3.26 \times 10^{-3}$ \\
Pyrogallol & $6.02 \times 10^{-3}$ & $7.46 \times 10^{-3}$ \\
Resorcinol & $1.19 \times 10^{-3}$ & $3.26 \times 10^{-3}$ \\
Hydroquinone & $4.75 \times 10^{-3}$ & $1.32 \times 10^{-2}$ \\
$p$-Aminophenol & $8.18 \times 10^{-2}$ & $1.11 \times 10^{-2}$ \\
$p$-Aminobenzoic & $1.19 \times 10^{-3}$ & $2.51 \times 10^{-2}$ \\
Adrenaline & $8.12 \times 10^{-2}$ & $7.11 \times 10^{-2}$ \\
Noradrenaline & $7.13 \times 10^{-3}$ & $6.51 \times 10^{-2}$ \\
\hline
\end{tabular}
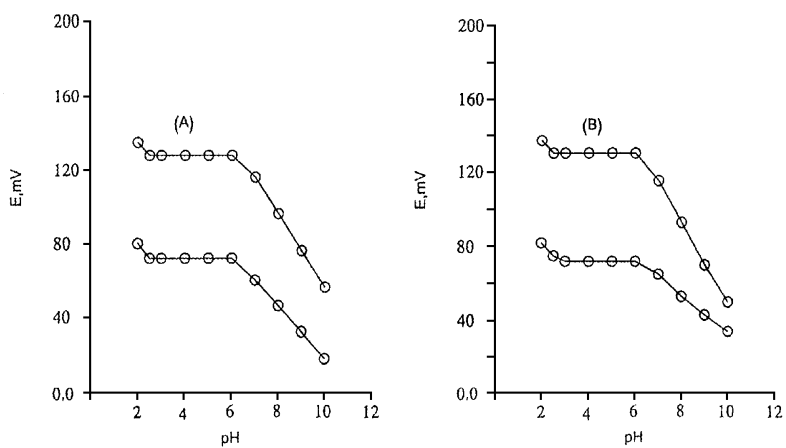

Fig. 3 Effect of the $\mathrm{pH}$ on the potential responses of dopamine sensors with CE-TPB (A) and CE-PTA (B).

cation size of the tested anions. Thus, dopamine hydrochloride can be shifted very well into the cavity of CE-PTA; this complex may be stronger than that of CE-TPB with dopamine. This effect leads to a higher concentration of complex species in the membrane with CE-PTA. Boles and Buck ${ }^{25}$ have reported that, at a high concentration of complex species in membranes, an anion response can be observed that results in a reduction of the cation response. Thus, upon using 12-crown-4, the Nernstian slope response seems to demonstrate that the mobility of the cation complex is much higher than that of the free anion in the membrane phase. The sensor CE-PTA was found to be better than the sensor CE-TPB in terms of calibration slope, the near-Nernstian response over a wide range of concentrations and the detection limit under the condition of solvent mediator $o$-NPOE. This behavior is most likely attributed to the high solubility of the complex ion associate CE-PTA, as compared to CE-TPB.

\section{Effect of the $\mathrm{pH}$}

Measurements of the $\mathrm{pH}$ dependence of dopamine sensors were performed over a wide range of $\mathrm{pH} 2-10$. The $\mathrm{pH}$ was adjusted using dilute sodium hydroxide and/or hydrochloric acid. The potential-pH profile for $10^{-2}$ and $10^{-3} \mathrm{M}$ drug 
Table 3 Determination of dopamine in some pharmaceutical formulations using direct potentiometry (A), spectrophotometry (B) and non-aqueous titrimetry $(\mathrm{C})$

\begin{tabular}{|c|c|c|c|c|}
\hline \multirow{3}{*}{ Pharmaceutical product } & \multicolumn{4}{|c|}{ Recovery, $\%^{\mathrm{a}}$} \\
\hline & \multicolumn{2}{|c|}{ A } & \multirow[t]{2}{*}{ B } & \multirow[t]{2}{*}{$\mathrm{C}$} \\
\hline & CE-PTA & CE-TPB & & \\
\hline Intropin, 200 mg/5 ml, Amp. Dupont, Co., UK & $100.3 \pm 0.7$ & $99.4 \pm 0.6$ & $98.3 \pm 0.8$ & $98.2 \pm 0.7$ \\
\hline Dopamine hydrochloride $200 \mathrm{mg} / 5 \mathrm{~mL}$, Amp. DBL, Co., UK & $99.5 \pm 0.6$ & $100.0 \pm 0.8$ & $98.1 \pm 0.7$ & $98.4 \pm 0.8$ \\
\hline Dopamine hydrochloride $100 \mathrm{mg} / 5 \mathrm{~mL}$, Amp. DBL, Co., UK & $99.9 \pm 1.2$ & $98.6 \pm 1.2$ & $99.3 \pm 0.9$ & $98.21 \pm 1.4$ \\
\hline Dopamine Fresenius 200 mg/5 ml, Amp. Fresenius Co., Germany & $99.6 \pm 0.5$ & $99.2 \pm 0.7$ & $97.9 \pm 0.9$ & $98.7 \pm 0.5$ \\
\hline
\end{tabular}

a. Average of three measurements \pm standard deviation.

solutions using the two sensors are summarized in Fig. 3. The data revealed a linear potential versus the $\mathrm{pH}$ in the range of $\mathrm{pH}$ $3-6$ from the point of view of sensor functions. It is apparent that, at $\mathrm{pH}<3$, the dopamine sensors became progressively more sensitive to the protonated dopamine species. In addition, the e.m.f readings sharply increased. In a test at $\mathrm{pH}>6$, precipitation of the free dopamine base occurred with a gradual increase in the concentration of the unprotonated species. ${ }^{18}$ It has been shown that more than $50 \%$ of dopamine hydrochloride doubles the charge and, consequently, the concentration of the unprotonated species gradually increases, and therefore the drug cations become sensible.

\section{Effect of foreign ions}

The potentiometric selectivity coefficient $\left(K_{\mathrm{DA}, \mathrm{B}}^{\mathrm{pot}}\right)$ of the CEPTA and the CE-TPB PVC dopamine membrane-based sensors depends on the selectivity of the ion-exchange process at the membrane-sample interface and the mobility of the respective ions in the membrane, as well as the hydrophobic interactions between the primary ions and the organic membrane. The selectivity of the dopamine membrane electrodes is also related to the free energy of transfer of the dopamine hydrochloride drug cation between the aqueous and organic phases. The potentiometric selectivity of the two sensors towards different substances was determined with $10^{-3} \mathrm{M}$ aqueous solutions of dopamine hydrochloride and foreign compounds using a separate solution method ${ }^{26,27}$ at $\mathrm{pH} 4.0$. The obtained results are summarized in Table 2. Good selectivity towards dopamine was achieved in the presence of many inorganic and organic cations, amines, quinons and phenols, pharmaceutical excipients and diluents commonly used in drug formulations (e.g. glucose, lactose, maltose) at concentrations as high as a 10-100 fold molar excess over the dopamine concentration. The CE-PTA sensor was found to be more selective than the CE-TPB sensor. The other substances listed in Table 2 were chosen as representative of potentially low-level contaminants in pharmaceutical preparations or in biological fluids.

\section{Potentiometric determination of dopamine in dosage forms}

The proposed CE-PTA and CE-TPB sensors were successfully applied for the analysis of dopamine in pure form and in pharmaceutical preparations. The results for the analysis of dopamine in pharmaceutical preparations by the developed sensors are summarized Table 3. The data obtained by the proposed sensors are in good agreement with the claimed values, and compare favorably with the standard method of British Pharmacopoeia. ${ }^{28}$ Average recoveries of 99.8 and $99.6 \%$ with mean standard deviations of $\pm 0.6 \%$ and $\pm 0.7 \%$, were obtained employing the CE-TPB and CE-PTA sensors, respectively. The data are also in good agreement with the certified values obtained by the reported spectrophotometric method $^{20}$ (average recovery of $98.1 \%$, and standard deviation of $\pm 0.8 \%$ ) and British Pharmacopoeia non-aqueous titrimetry ${ }^{29}$ (89.4 and standard deviation \pm 0.6 ). It is obvious from the results given in Table 3 that the proposed sensors can be successfully used for the selective determination of dopamine hydrochloride in the presence of its degradations.

Alternatively, the standard addition method was also applied to the analysis of dopamine in tablets by the proposed sensors. Good correlations of 0.998 and a recovery percentage of $99.7 \pm$ $0.6 \%$ were obtained between the experimental results and the claimed values. The two sensors are also suitable for the quality-control analysis of dopamine in tablets without any prior pretreatment.

The prepared sensors were successfully used for the analysis of dopamine hydrochloride in pharmaceutical preparations at levels $>1.9 \mu \mathrm{g} \mathrm{ml}^{-1}$. The developed sensors are selective, sensitive and attractive in terms of low cost for routine control analysis. The proposed sensors exhibit favorable performance characteristics of $\mathrm{pH}$, low detection limit, calibration slope, and fast response; also the drug does not need any pretreatment or separation step and yields highly selective measurements in a synthetic mixture of electroactive substances. The sensors can be produced simply and reliably on a large scale and for these reasons may be regarded as being disposable.

\section{References}

1. T. Stabler, J. Med. Tech., 1986, 3, 351.

2. J. Axelord, J. Neurosurg., 1981, 55, 669.

3. C. L. Guan, J. Ouyang, Q. L. Li, B. H. Liu, and W. R. G. Baeyens, Talanta, 2000, 50, 1197.

4. J. Liu, Z. H. Wang, G. A. Luo, Q. W. Li, and H. W. Sun, Anal. Sci., 2002, 18, 751.

5. P. Nagaraja, K. C. S. Murthy, K. S. Rangappa, and N. M. M. Gowda, Talanta, 1998, 46(8), 39.

6. C. L. Hryncewioz, M. Koberda, and M. S. Konkowski, Electroanalysis, 1998, 7, 477.

7. V. A. Cardon, A. M. Mauri, E. M. Llobat, M. C. Pascual, and M. J. Simeon, Fresenius, J. Anal. Chem., 1994, 350(12), 706.

8. M. E. El-Kommos, F. A. Mohamed, and A. S. Kheder, J. Assoc. Anal. Chem., 1990, 73, 516.

9. R. M. V. Camanas, J. M. S. Mallois, J. R. T. Lapasio, and G. Ramisramos, Analyst, 1995, 120, 1767.

10. E. Hollenbach, C. Schultz, and H. Lehnert, Life Sci., 1998, 63, 737.

11. O. Niwa and M. Morita, Anal. Chem., 1996, 68, 737. 
12. M. Takahashi, M. Morita, O. Niwa, and H. Tabei, Sens. Actuators, 1993, B13, 336.

13. L. R. Junior, J. C. B. Fernandes, G. De Oliveira Neto, and L. T. Kubota, J. Electroanal. Chem., 2000, 481, 34.

14. M. Chen and H. L. Li, Electroanalysis, 1998, 7, 477.

15. O. Niwa, M. Morita, and H. Tabei, Sens. Actuators, 1993, B14, 558 .

16. K. Odashima, K. Yagi, K. Tohda, and Y. Umezawa, Bioinorg. Med. Chem. Lett., 1999, 9, 2375.

17. E. S. Forzani, G. A. Rivas, and V. M. Solis, J. Electroanal. Chem., 1997, 435, 77.

18. T. S. Ma and S. S. M. Hassan, "Organic Analysis Using Ion Selective Electrodes", 1982, Vols. 1 and 2, Academic Press, London.

19. A. Craggs, G. J. Moody, and J. D. R. Thomas, J. Chem.
Educ., 1974, 51, 451.

20. M. E. El-Kommos, J. Pharm. Belg., 1987, 42, 371.

21. C. J. Pederson, J. Am. Chem. Soc., 1967, 89, 7017.

22. D. DaWang and J. S. Shih, Analyst, 1985, 110, 635.

23. J. Jeng and J. S. Shih, Analyst, 1984, 109, 641.

24. IUPAC Analytical Chemistry Division, Pure and Appl. Chem., 1995, 67, 507.

25. J. H. Boles and R. P. Buck, Anal. Chem., 1972, 45, 2057.

25. S. S. M. Hassan and N. M. H. Rizk, Anal. Lett., 2000, 33, 1037.

26. N. M. H. Rizk, Mikrochim. Acta, 2002, 138, 53.

27. British Pharmacopoeia, 2000, Vol. 1, Version 4.0 Crown Copyright.

28. H. Zhoo, Y. Zha, and Z. Yuan, Anal. Chim. Acta, 2001, $441,117$. 